\title{
Abnormal brain activation in excoriation (skin-picking) disorder: evidence from an executive planning fMRI study
}

\author{
Brian L. Odlaug, Adam Hampshire, Samuel R. Chamberlain and Jon E. Grant
}

\section{Background}

Excoriation (skin-picking) disorder (SPD) is a relatively common psychiatric condition whose neurobiological basis is unknown

\section{Aims}

To probe the function of fronto-striatal circuitry in SPD.

\section{Method}

Eighteen participants with SPD and 15 matched healthy controls undertook an executive planning task (Tower of London) during functional magnetic resonance imaging (fMRI). Activation during planning was compared between groups using region of interest and whole-brain permutation cluster approaches.

\section{Results}

The SPD group exhibited significant functional underactivation in a cluster encompassing bilateral dorsal striatum (maximal in right caudate), bilateral anterior cingulate and right medial frontal regions. These abnormalities were, for the most part, outside the dorsal planning network typically activated by executive planning tasks.

\section{Conclusions}

Abnormalities of neural regions involved in habit formation, action monitoring and inhibition appear involved in the pathophysiology of SPD. Implications exist for understanding the basis of excessive grooming and the relationship of SPD with putative obsessive-compulsive spectrum disorders.

\section{Declaration of interest}

B.L.O. has consulted for H. Lundbeck A/S. S.R.C. consults for Cambridge Cognition. J.E.G. has received research grants from the National Center for Responsible Gaming, Forest, Transcept, Roche and Psyadon Pharmaceuticals.

\section{Copyright and usage}

(c) The Royal College of Psychiatrists 2016.
Excoriation (skin-picking) disorder (SPD), also known as pathological skin-picking, neurotic/psychogenic excoriation and dermatillomania, is a body-focused repetitive behaviour characterised by compulsive picking of skin causing tissue damage. ${ }^{1}$ As a result of the growing body of research and recognition of SPD as a clinically significant condition over the past $10-15$ years, SPD is now included in DSM-5 under the name 'excoriation (skin-picking) disorder' as a full disorder for the first time. ${ }^{2}$ SPD is associated with a significant degree of psychosocial dysfunction, poor quality of life and medical complications ${ }^{3-4}$ and appears to be a fairly common disorder in the general population. In recent years, prevalence studies have indicated rates of SPD between 1.2 and $5.4 \%$ in population samples. ${ }^{5-8}$ From a phenomenological perspective, SPD appears similar to trichotillomania and onychophagia (compulsive nail-biting), in that it is characterised by repetitive and excessive maladaptive grooming habits that are difficult for individuals to suppress. ${ }^{1}$ Indeed, these different types of symptoms commonly co-occur within individuals, leading to the notion that they be considered body-focused repetitive behaviours, which may share a common pathophysiological basis. ${ }^{9}$ Another somewhat complementary perspective is that SPD be considered an obsessive-compulsive 'spectrum' disorder or disorder of the 'impulsive-compulsive spectrum' given the overlapping phenomenological and clinical characteristics of SPD and obsessive-compulsive disorder (OCD) and high rate of co-occurrence between the two disorders. In a study conducted in 901 patients with OCD, for example, $16.4 \%$ of the sample met criteria for concomitant SPD, while $4.9 \%$ met criteria for concomitant trichotillomania. ${ }^{10}$ Recent aetiological research examining proposed genetic and environmental risk factors for the development of the obsessive-compulsive spectrum disorders found a high rate of overlap between these disorders, especially SPD and trichotillomania. ${ }^{11}$ Therefore, the relationship between SPD and OCD may be particularly pertinent in terms of such a conceptualisation.

Little is known regarding the neurobiological mechanisms or constructs involved in the aetiology of SPD. Recent structural imaging of SPD indicated reduced fractal anisotropy in tracts distributed bilaterally, which included white matter close to the anterior cingulate cortices, compared with controls. ${ }^{12}$ These regions were remarkably similar to those separately found to be abnormal in trichotillomania. ${ }^{13,14}$ For OCD, there exists a large body of literature, implicating dysregulation of the striatum (involved in habit generation) coupled with a lack of top-down input from cortical regions (including both medial and lateral prefrontal regions) responsible for various cognitive processes. ${ }^{15}$ Patients with OCD often show behavioural impairments in executive planning (see for example Chamberlain et al, Tükel et al and Kashyap et $\left.a l^{16-18}\right)$. Such deficits extend to relatives of patients with OCD who are clinically asymptomatic, highlighting the centrality of this cognitive function and its implicated neural substrates in the pathophysiology - it may well represent an intermediate biological 'vulnerability marker' for OCD. ${ }^{19,20}$ As such, planning-related functional magnetic resonance imaging (fMRI) tasks represent a useful means of probing frontostriatal integrity in OCD and related conditions, since they challenge salient distributed neural circuitry. Decreased responsiveness in the (mainly dorsolateral) prefrontal cortex and caudate nucleus was found during fMRI executive planning tasks in people with OCD compared with controls. ${ }^{21}$

The objective of this study was to probe the integrity of fronto-striatal circuitry in people with SPD compared with 
matched healthy controls using an fMRI executive planning paradigm. We hypothesised that SPD would be associated with underactivation during planning in the striatum and (dorsolateral) prefrontal cortex $v$. controls, supporting a neurobiological relationship between SPD and OCD.

\section{Method}

\section{Study participants}

Men and women aged 18-54 with a primary diagnosis of SPD based on DSM-5 criteria and a structured clinical interview with a board-certified psychiatrist with expertise in SPD and bodyfocused repetitive behaviours were recruited by newspaper and poster advertisements. All participants were recruited and underwent neuroimaging procedures at the University of Minnesota Medical Center.

Inclusion criteria included: (a) participants met DSM-5 criteria for SPD for at least the past 12 consecutive months: (i) recurrent skin-picking resulting in skin lesions; (ii) repeated attempts to decrease or stop skin-picking; (iii) picking causes clinically significant distress or impairment in social, occupational or other important areas of functioning; (iv) picking is not because of the direct physiological effects of a substance (for example (meth)amphetamine, cocaine) or a general medical condition (such as scabies); and (v) skin-picking is not restricted to the symptoms of another mental disorder (such as skin-picking because of fixed beliefs about skin infestation in delusional disorder or parasitosis, preoccupation with appearance in body dysmorphic disorder); (b) a minimum score of $>16$ on the Yale Brown Obsessive Compulsive Scale modified for Neurotic Excoriation (NE-YBOCS);22,23 and (c) picking behaviour occurred daily for at least 30 min consistently over the past 12 months.

Exclusion criteria in those with SPD comprised: (a) unstable medical illness or clinically significant abnormalities on physical examination; (b) current pregnancy or lactation; (c) lifetime history of bipolar disorder type I or II, dementia or any psychotic disorder; (d) any current (past 12 months) DSM-5 psychiatric disorder including OCD, body dysmorphic disorder, trichotillomania, gambling disorder, nicotine dependence and disruptive, impulse control and conduct disorders; (e) initiation of psychotherapy or pharmacotherapy within 3 months prior to study entry and, if taking medication, no dose changes for the preceding 3 months; (f) history of head injury or neurologic disorders; and $(\mathrm{g})$ any contraindications to MRI based on safety screening and clinical history. In addition, no participants had a history of hypertension or diabetes, conditions which may interfere with brain imaging.

After a complete description of the study to the participants, written informed consent was obtained. No secondary consents were allowed for the study (i.e. parent/guardian consent was not allowed). A full institutional review board approved the consent procedures and all study procedures were carried out in accordance with the ethical principles for medical research involving human participants established in the 2008 Declaration of Helsinki. Age- and gender-matched healthy controls were recruited via word of mouth, poster and newspaper advertisements. All control group participants were free of any lifetime psychiatric disorder according to the Structured Clinical Interview for DSM-IV (SCID-I) ${ }^{24}$ and DSM-5 criteria for SPD.

\section{Assessments}

Participant interviews and scale administration were conducted by a board-certified psychiatrist with expertise in the assessment and treatment of body-focused repetitive behaviours, OCD and disorders of impulse control. Psychiatric comorbidity was assessed using the SCID-I. The severity of SPD was assessed using the NE-YBOCS. All participants underwent a safety screening for contraindication for MRI at study entry and at the scanning facility to ensure safety.

Functional imaging was completed within 7 days of the intake assessment. We conducted one fMRI task based on our desire to probe the integrity of striatal and frontal lobe function in SPD. As such, the Tower of London task of frontal lobe integrity was chosen given that this paradigm had previously been found to be sensitive to brain dysfunction in those with gambling disorder, ${ }^{25,26}$ obsessive-compulsive disorder, ${ }^{20}$ Parkinson's disease, ${ }^{27,28}$ and National Football League players with executive deficits. ${ }^{29}$ Given significant time and funding constraints, this task was chosen based on our hypotheses of executive function deficits in the SPD group and this previous research. The Tower of London task has been validated in other clinical contexts but never before applied to SPD. ${ }^{28,30}$ The task lasted for approximately $10 \mathrm{~min}$, during which planning and subtracting problems were shown on a computer screen (inter-trial interval jittered 5-15s). For each trial, participants were presented with two sets of tubes, with a random assortment of three coloured balls inserted in the tubes. Participants were prompted with a cue screen displaying the word 'plan' or 'subtract' before each new problem was presented. For 'plan' trials, participants were instructed to mentally determine the minimal number of ball moves required to make the top set of tubes match the bottom set of tubes. Participants indicated their response using a button-box placed in their right hand by pressing one of four buttons numbered 1-4; as such, trial duration was response driven (for further details see Williams-Gray et $a^{28}$ ). For 'subtract' trials, participants were instructed to count the number of balls in the top row and subtract them for the number of balls in the lower row. Again, responses were indicated using a button-box. Both tasks used only problems with correct responses of 2, 3 or 4 . Participants were not provided with feedback regarding the correctness of their responses in order to minimise any possible influence of error-related feedback on brain activation. Task difficulty varied via a predefined pseudo-randomised sequence. All participants underwent a training session before scanning to ensure that they understood the rules of the task and were able to perform it adequately, and to minimise the risk of betweengroup behavioural differences in the scanner, which can represent major confounds in interpreting fMRI group differences.

All participants were scanned at the University of Minnesota using a 3-Tesla Siemens (Munich, Germany) TIM Trio MRI scanner. In total $330 T_{2}$-weighted echo-planar images depicting blood oxygen level-dependent (BOLD) signal were acquired, with the first 10 being discarded to avoid $T_{1}$-equilibrium effects. The fMRI sequence data were as follows: acquisition time $2 \mathrm{~s}$ (per volume); type: 2D; slices: 32 ; slice gap: $25 \%$; slice thickness: $3 \mathrm{~mm}$; slice order: descending $(32,31, \ldots, 1)$; field of view: $192 \times 192 \mathrm{~mm}$; matrix: $64 \times 64$; resolution: $3 \times 3 \mathrm{~mm}$; repetition time: $2000 \mathrm{~ms}$; echo time: $30 \mathrm{~ms}$; flip angle: $78^{\circ}$; bandwidth: $2232 \mathrm{~Hz} / \mathrm{Px}$; echo spacing: $0.51 \mathrm{~ms}$.

\section{Data analysis}

Potential differences between the study groups on demographic and clinical measures of interest were explored using independent sample $t$-tests or alternative non-parametric tests as indicated, with significance defined as $P<0.05$ uncorrected for these purposes. Imaging data were pre-processed using the methods as previously reported. $^{28}$ In brief, the scan data were motion-corrected, slice-time acquisition corrected, co-registered to structural scans 
(standard MPRAGE), and normalised to the standard Montreal Neurological Institute echo-planar imaging template. The resulting images were smoothed with a $8 \mathrm{~mm}$ full-width at halfmaximum Gaussian kernel. We first examined whether the task activated the expected frontostriatal planning network by conducting a whole brain analysis using SPM (version 5) for the plan minus subtract contrast, collapsing across all study participants $(P<0.05$ false discovery rate-corrected). The general linear model included the onsets and durations of the task events convolved with the canonical haemodynamic response function. It also included movement parameters and a constant term. There has been some debate in the neuroimaging field regarding the choice of FDR $v$. the alternative family-wise error-correction method for multiple comparisons (for discussion see for example Bennett et $a l^{31}$ ). Our choice of FDR correction was in order to avoid the elevated risk of false positives if using family-wise error, and in order to maintain consistency of approach with prior work (for example Williams-Gray et al and Hampshire et $a l^{28,29}$ ).

In order to explore potential differences between the groups in terms of fMRI activation, we used the contrast of task minus rest (since this typically yields greater variance for detection of crossgroup effects and is more easily interpretable for such analyses, as compared with the count minus subtract contrast). We used two complementary analysis approaches.

\section{Select region of interest (ROI) analysis}

Mean activation data were extracted from the activation clusters and compared cross-group using the MarsBaR Regions of Interest toolbox (random effects). ${ }^{32}$ Regions of interest were defined as $10 \mathrm{~mm}$ spheres based on peak activation coordinates derived from an independent healthy volunteer data-set (the first 50 healthy controls participating in Hampshire et $a l^{29}$ ); note that this dataset was entirely independent with no participants taking part in both studies. The region of interest (ROI) coordinates were: dorsolateral prefrontal cortex, left $-22,14,56$; dorsolateral prefrontal cortex, right 26, 26, 56; posterior parietal cortex, left $-36,-76,38$; posterior parietal cortex, right $44,-70,34$; frontoparietal cortex, right $28,50,2$. MarsBaR is a particularly sensitive approach when there is a strong prior hypothesis, because it averages values from all voxels within the ROI. This eschews the need to correct for many voxel-wise comparisons and thereby increases the sensitivity for detecting positive effects, mitigating the risk of false negatives.

\section{Whole-brain unconstrained analysis}

Cross-group differences were examined using robust permutation modelling (Cambridge Brain Analysis Software (CamBA), random effects). To provide stringent control for multiple comparisons, cluster correction was applied such that the expected number of false-positive clusters for the contrast of interest was less than one. Unlike parametric methods (for example those used in SPM), permutation modelling using CamBA is robust against outliers and non-normally distributed data. Moreover, CamBA offers a more advanced correction approach than SPM. More specifically, it uses cluster weights as opposed to extents and controls for multiple comparisons at the cluster level with permutation modelling.

We also examined correlations between clinical severity using the NE-YBOCS and (a) behavioural task measures; and (b) extracted mean ROI and cluster values from the imaging analyses (Pearson's $r$, uncorrected $P<0.05$ ). Correlations were also conducted between age at symptom onset and these measures. Exploratory $t$-tests (uncorrected $P<0.05$ ) were used to explore the possible moderating influence of medication status and lifetime history of other psychiatric disorders in the SPD individuals, with respect to disease severity and any behavioural or brain activation measures that differed between groups.

\section{Results}

\section{Participant characteristics}

In total, 18 participants (mean age 29.9 years, s.d. $=9.7,100 \%$ women) with SPD and 15 age-matched controls (mean age 32.9 years, s.d. $=14.7,86.7 \%$ women) met inclusion criteria and underwent the clinical assessment and functional neuroimaging (Table 1). The SPD group had a mean age at onset of 11.3 years (s.d. $=4.1)$ and a mean duration of illness of 18.6 years (s.d. $=9.1$ ) years. A total of nine $(50 \%)$ participants in the SPD group met DSM-5 criteria for another lifetime psychiatric condition: $n=6$ major depressive disorder; $n=2$ major depressive disorder and other specified anxiety disorder; $n=1$ generalised anxiety disorder. Per our exclusion criterion, however, none of the participants had a current psychiatric disorder, other than SPD. Five participants in this group were taking stable doses of psychotropic medications at the time of imaging: three were taking a selective serotonin reuptake inhibitor (SSRI) and two were taking a selective noradrenergic reuptake inhibitor (SNRI). Of the three participants on an SSRI, one was taking citalopram

\begin{tabular}{|c|c|c|c|c|}
\hline \multirow[b]{2}{*}{ Variable } & \multicolumn{2}{|c|}{ Baseline } & \multirow[b]{2}{*}{$t$-test } & \multirow[b]{2}{*}{$P$} \\
\hline & SPD group $(n=18)$ & Control group $(n=15)$ & & \\
\hline Age, years: mean (s.d.) & $29.9(9.7)$ & $32.9(14.7)$ & 0.703 & 0.488 \\
\hline Gender, female, $n(\%)$ & $18(100)$ & $13(86.7)$ & & $0.199^{\mathrm{a}}$ \\
\hline White, $n(\%)$ & $17(94.4)$ & $12(80)$ & & $0.308^{\mathrm{a}}$ \\
\hline Marital status, single, $n(\%)$ & $13(72.2)$ & $10(66.7)$ & & $1.000^{\mathrm{a}}$ \\
\hline $\begin{array}{l}\text { Education, } n(\%) \\
\text { Less than a college degree } \\
\text { College degree or higher }\end{array}$ & $\begin{array}{r}7(38.9) \\
11(61.1)\end{array}$ & $\begin{array}{r}3(20) \\
12(80)\end{array}$ & & $0.283^{a}$ \\
\hline Right handedness, $n$ (\%) & $15(83.3)$ & $13(86.7)$ & & $1.000^{\mathrm{a}}$ \\
\hline Age at onset, years: mean (s.d.) & $11.3(4.1)$ & - & & \\
\hline Any lifetime psychiatric comorbidity, $n$ (\%) & $9(50)$ & - & & \\
\hline $\begin{array}{l}\text { Yale Brown Obsessive Compulsive Scale Modified for Neurotic Excoriation, } \\
\text { total score: mean (s.d.) }\end{array}$ & $21.9(4.6)$ & - & & \\
\hline
\end{tabular}


augmented with aripiprazole and another was taking citalopram augmented with buspirone. Of the two participants on an SNRI, this was augmented with bupropion for one participant and with lamotrigine for the other.

No significant demographic or clinical severity differences were found between those in the SPD group with a history of a lifetime psychiatric disorder and those without such a history (all $P>0.010$ ) (age: $P=0.641$, age at onset: $P=0.507$, NE-YBOCS: $P=0.243$ ) or between those taking a psychotropic medication $v$. those not taking medication (age: $P=0.108$, age at onset: $P=0.510$, NE-YBOCS: $P=0.569$ ).

\section{Imaging results}

No individually clinically significant MRI structural abnormalities were identified. On the Tower of London task, the SPD group did not differ significantly from the control group in terms of response times (subtract trials: SPD group mean $3.6 \mathrm{~s}$ (s.d. $=0.8)$, control group mean 4.9 s $($ s.d. $=2.8), P=0.10$; plan trials: SPD group mean $8.4 \mathrm{~s}($ s.d. $=3.0)$, control group $8.7 \mathrm{~s}$ (s.d. $=2.5), P=0.73$ ). Similarly, the SPD and control groups did not differ significantly in terms of percentage correct on the task (subtract trials: SPD group mean $82.5 \%$ (s.d. $=11.0 \%$ ), control group mean $71.7 \%$ (s.d. $=17.7 \%), P=0.05$; plan trials: SPD group mean $74.9 \%$ (s.d. $=19.5 \%)$, control group mean $70.2 \%$ (s.d. $=20.4 \%), P=0.51$ ).

As expected, the Tower of London task activated a network of brain regions during executive planning, including frontal, parietal and striatal brain regions based on the plan minus subtract contrast. In close concordance with previous studies that have used this paradigm, subtracting trials from planning trials rendered a dorsal subset of these regions including dorsolateral prefrontal cortex and posterior parietal cortex (Table 2 and online Fig. DS1). In the ROI (MarsBaR) analysis, no significant activation differences were evident between the two groups within the dorsal planning network (Table 3, $P>0.05$ uncorrected; Fig. 1).

Whole brain analysis indicated that SPD was associated with significant underactivation during the task, compared with the control group, in a single cluster (Table 4 and online Fig. DS2). The cluster was maximal in the right caudate nucleus $(14,24$, -8 ), and included the bilateral caudate, right putamen, bilateral anterior cingulate cortices, bilateral olfactory lobes, and right frontal (superior medial and middle orbital) regions. In exploratory

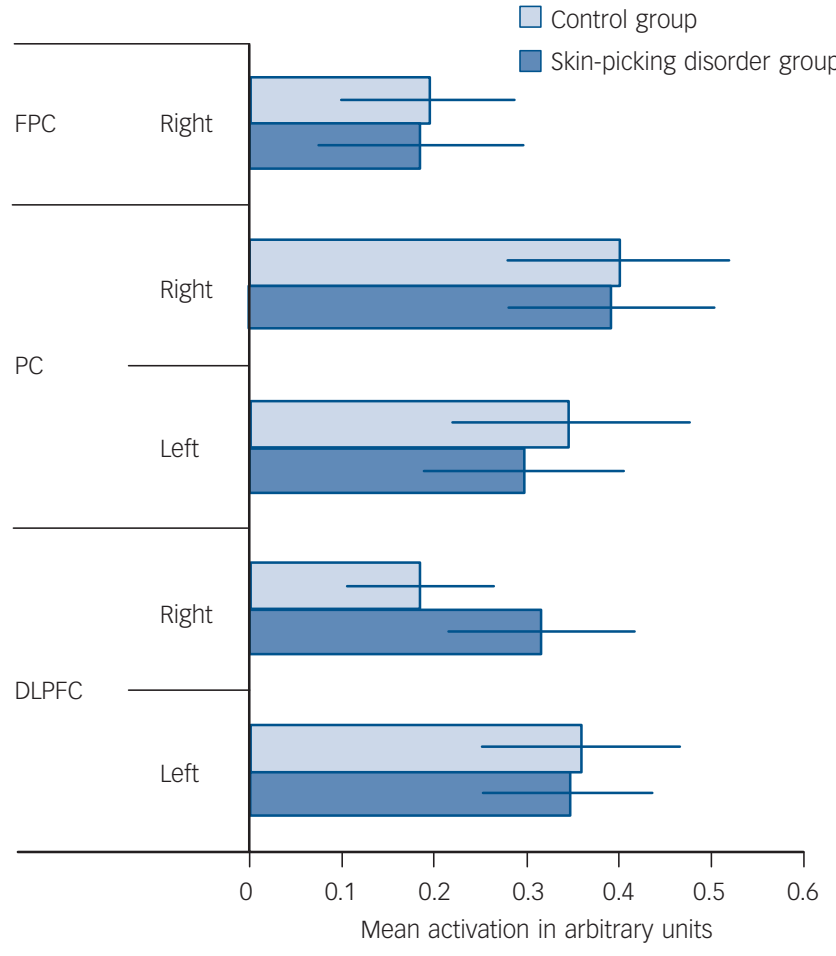

Fig. 1 Tower of London task: task minus rest collapsed across difficulty level.

FPC, frontoparietal cortex right; PC, parietal cortex; DLPFC, dorsolateral prefrontal cortex.

analyses, mean activation in the identified brain cluster did not differ significantly as a function of medication status $(P=0.676)$ nor as a function of past history of psychiatric comorbidities in the SPD group $(P=0.702)$.

No significant correlation was found between mean brain activation in the identified cluster and task behavioural measures $(P=0.603)$; nor between NE-YBOCS total scores and task behavioural measures (both $P>0.10$ ). Furthermore, no significant correlation was identified between mean brain activation in the identified cluster and NE-YBOCS total scores $(P=0.211)$ nor age at symptom onset $(P=0.542)$.

\begin{tabular}{|c|c|c|c|c|c|}
\hline \multirow[b]{2}{*}{ Cluster, equivk } & \multirow[b]{2}{*}{$\begin{array}{l}\text { Cluster, } P \text { (family-wise } \\
\text { error-corrected) }\end{array}$} & \multicolumn{3}{|c|}{ Peak } & \multirow[b]{2}{*}{$\begin{array}{c}\text { MNI coordinates, mm: } \\
\qquad x, y, z\end{array}$} \\
\hline & & $T$ & equivz & $\begin{array}{l}P \text { (false discovery } \\
\text { rate-corrected) }\end{array}$ & \\
\hline \multirow[t]{3}{*}{800} & $<0.001$ & 7.72 & 6.69 & $<0.001$ & $0,-60,45$ \\
\hline & & 5.31 & 4.92 & $<0.001$ & $18,-54,21$ \\
\hline & & 4.74 & 4.45 & 0.001 & $3,-60,30$ \\
\hline \multirow[t]{3}{*}{175} & 0.002 & 6.62 & 5.92 & $<0.001$ & $-36,-81,39$ \\
\hline & & 3.82 & 3.66 & 0.007 & $-42,-75,21$ \\
\hline & & 3.69 & 3.54 & 0.009 & $-21,-81,48$ \\
\hline 192 & 0.001 & 5.3 & 4.91 & $<0.001$ & $-24,6,57$ \\
\hline 55 & 0.204 & 5.26 & 4.87 & $<0.001$ & $-15,-57,21$ \\
\hline \multirow[t]{3}{*}{362} & $<0.001$ & 5.02 & 4.68 & $<0.001$ & $42,-48,27$ \\
\hline & & 4.76 & 4.46 & 0.001 & $48,-66,30$ \\
\hline & & 4.74 & 4.45 & 0.001 & $51,-54,21$ \\
\hline \multirow[t]{3}{*}{353} & $<0.001$ & 4.91 & 4.59 & $<0.001$ & $27,12,51$ \\
\hline & & 4.8 & 4.5 & 0.001 & $24,3,57$ \\
\hline & & 4.35 & 4.12 & 0.002 & $33,30,48$ \\
\hline
\end{tabular}


Table 3 Region of interest (ROI) approach (Marsbar): comparison of activation between the skin-picking disorder group and control group within the frontoparietal network, task minus rest contrast

\begin{tabular}{|lrcc|}
\hline ROI (MNI coordinates) & Contrast value & $t$ statistic & Uncorrected $P$ \\
\hline Left dorsolateral prefrontal cortex $(-22,14,56)$ & 0.04 & 0.32 & 0.375 \\
\hline Right dorsolateral prefrontal cortex $(26,26,56)$ & -0.009 & -0.67 & 0.746 \\
\hline Left posterior parietal cortex $(-36,-76,38)$ & 0.04 & 0.23 & 0.409 \\
\hline Right posterior parietal cortex $(44,-70,34)$ & -0.03 & -0.16 & 0.563 \\
\hline Right frontoparietal cortex $(28,50,2)$ & 0.05 & 0.36 & 0.359 \\
MNI, Montreal Neurological Institute. & & & \\
\hline
\end{tabular}

\begin{tabular}{|c|c|c|}
\hline & MNI coordinates & Voxels \\
\hline Cluster 1 & & 1607 \\
\hline Caudate_R, max -3.937232 & $14,24,-8$ & 72 \\
\hline BA6: Frontal_Sup_Orb_R & & 17 \\
\hline BA21: Olfactory_L & & 18 \\
\hline BA22: Olfactory_R & & 69 \\
\hline BA24: Frontal_Sup_Medial_R & & 6 \\
\hline BA26: Frontal_Mid_Orb_R & & 39 \\
\hline BA28: Rectus_R & & 83 \\
\hline BA31: Cingulum_Ant_L & & 9 \\
\hline BA32: Cingulum_Ant_R & & 38 \\
\hline BA71: Caudate_L & & 123 \\
\hline BA72: Caudate_R & & 256 \\
\hline BA74: Putamen_R & & 32 \\
\hline Space outside regions & & 917 \\
\hline
\end{tabular}

Brennan et $\left.a l^{33}\right)$. There is an ongoing search in psychiatry for models of the neurobiological circuitry implicated in given disorders. Greater understanding of such circuitry is likely to have ramifications for novel treatments and more appropriate diagnostic classification systems. ${ }^{34,35}$ Although caution is warranted when comparing findings across studies that have used somewhat different methodologies, it is interesting to contrast the intact planning performance we observed in SPD coupled with normal activation in the dorsal planning network according to the ROI analysis, to previous findings of deficient executive planning and hypoactivation in this network in patients with OCD $v$. controls. ${ }^{21}$ Viewed together, these results militate against a primary planning deficit in SPD. Rather, the data suggest that SPD is associated with abnormal function of neural regions outside the usual 'planning' network: specifically, as indicated by the permutation cluster analysis, hypoactivation of the dorsal striatum, anterior cingulate cortices and right frontal regions. These regions are anatomically close to those found to be structurally abnormal in previous SPD and also trichotillomania research. ${ }^{12-14}$ Thus, it appears that SPD is associated not only with structural but also functional brain abnormalities in regions involved in habit generation, action monitoring and top-down inhibitory control processes. ${ }^{12}$ In OCD, dysfunction extends from medial to lateral prefrontal networks whereas in SPD, dysfunction may be more restricted to medial sectors of this circuitry.

These results suggest that cognitive fMRI tasks germane to other cognitive processes, such as motor inhibition and habit generation, may shed more light on the pathophysiology of SPD than executive planning tasks. In terms of the state $v$. trait nature of the currently identified neural abnormalities, we could not detect a significant correlation between activation and disease severity. The study may have been too small to detect such a relationship; alternatively, the hypoactivation could be 'trait' in nature: i.e. it may exist in people at risk of SPD even before symptoms develop. This lack of relationship between fMRI measures and disease severity could also theoretically be a consequence of the severity scale measure used. Since the NE-YBOCS (although a standard tool for measuring symptom severity for this disorder) only assesses severity over the past 7 days, it may not be a good reflection of more of a 'trait' cognitive dysfunction that arguably underlies SPD. Future work could address this issue by utilising an 'endophenotyping' design in which patients are recruited along with clinically unaffected first-degree relatives. minus rest), SPD was associated with significant underactivation in distributed neural circuitry including the bilateral dorsal striatum, bilateral anterior cingulate and right frontal regions. These neural abnormalities appeared to be unrelated to symptom severity or age at symptom onset.

\section{Comparison with findings from other studies}

Brain imaging has been helpful in furthering our understanding of the potential neurobiological mechanisms involved in other, similar compulsive behaviours such as OCD (see for example

\section{Limitations}

The demographics, clinical characteristics and severity of our SPD sample accord well with the characteristics of previously reported samples of patients with $\mathrm{SPD},{ }^{3}$ suggesting that our sample and findings may be representative of the female SPD population at large. However, several limitations to this current study should be considered. First, the relatively small sample size of both groups 
limits the power to make any definitive statements about neural processing in SPD. As a result of the limited sample size, we opted for the dual complementary approaches of utilising both an ROI analysis in conjunction with FDR correction (to maximise power and minimise type II error) and a separate whole brain permutation cluster analysis (to evaluate potential abnormalities in an unconstrained fashion, but at potential risk of diminished power). The cluster identified in the permutation analysis appeared to contain some white matter, a finding not uncommon in fMRI studies more broadly (see Gawryluk et $a l^{36}$ for discussion). This could be because of imprecision in the localisation of fMRI signal (particularly with gap slices) in that the regions could nonetheless represent grey matter activation differences; alternatively they may truly reflect abnormal activation associated with white matter, there being evidence elsewhere of structural white matter abnormalities in SPD. ${ }^{12}$ White matter findings using fMRI are, however, controversial and potentially problematic to interpret. Second, half of our sample had a lifetime psychiatric history and nearly one-third was taking a stable dose of a psychotropic medication at the time of imaging. This study was neither designed nor powered to address possible influences of medications and comorbidities on brain activation. Nonetheless, exploratory analyses showed that these variables did not significantly have an impact on brain activation in the identified cluster in the SPD group. Future studies will need to formally address this issue with larger sample sizes before firm conclusions are drawn about the observed abnormal activation patterns being entirely attributable to disease rather than potential confounds. Given that people with SPD report high rates of psychiatric comorbidity, ${ }^{1,4}$ however, we felt that excluding these participants would result in a less-generalisable sample than the SPD population at-large, as well as diminishing power for an already relatively small study for which funding is scarce. Finally, as the control group were, for the most part, recruited in advance of the SPD group, by chance two control participants were male, whereas none of the SPD group were male (and we had anticipated some males in the SPD group). Analysis confirmed, however, that inclusion of these two male controls did not affect the imaging results, in that the SPD-control group difference in activation in the identified cluster remained highly significant even when both male controls were excluded.

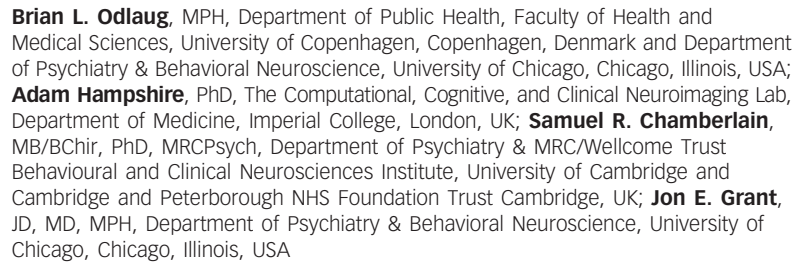

Correspondence: Brian L. Odlaug, MPH, Department of Public Health, Faculty of Health \& Medical Sciences, University of Copenhagen, Øster Farimagsgade 5 , DK-1353 Copenhagen K, Denmark. Email: brod@sund.ku.dk

First received 1 Mar 2014, final revision 25 Nov 2014, accepted 25 Nov 2014

\section{Funding}

This research was supported by a research grant from the Trichotillomania Learning Cente (B.L.O.), a Center for Excellence in Gambling Research grant by the National Center for Responsible Gaming (J.E.G.), an American Recovery and Reinvestment Act (ARRA) Grant from the National Institute on Drug Abuse (1RC1DA028279-01, .E.G.) and a starter grant for clinical lecturers from the Academy of Medical Sciences, UK (S.R.C.)

\section{References}

1 Grant JE, Odlaug BL, Chamberlain SR, Keuthen NJ, Lochner C, Stein DJ. Skin picking disorder. Am J Psychiatry 2012; 29: 1025-31.
2 American Psychiatric Association. Diagnostic and Statistical Manual of Mental Disorder (5th edn) (DSM-5). APA, 2013.

3 Odlaug BL, Grant JE. Clinical characteristics and medical complications of pathological skin-picking. Gen Hosp Psychiatry 2008; 30: 61-6.

4 Tucker BT, Woods DW, Flessner CA, Franklin SA, Franklin ME. The skinpicking impact project: phenomenology, interference, and treatment utilization of pathological skin-picking in a population-based sample. J Anxiety Disord 2011; 25: 88-95.

5 Hayes SL, Storch EA, Berlanga L. Skin picking behaviors: an examination of the prevalence and severity in a community sample. J Anxiety Disord 2009; 23: 314-9.

6 Keuthen NJ, Koran LM, Aboujaoude E, Large MD, Serpe RT. The prevalence of pathological skin-picking in US adults. Compr Psychiatry 2010; 51: 183-6.

7 Monzani B, Rijsdijk F, Cherkas L, Harris J, Keuthen N, Mataix-Cols D. Prevalence and heritability of skin-picking in an adult community sample: a twin study. Am J Med Genet B Neuropsychiatr Genet 2012; 159: 605-10.

8 Odlaug BL, Lust K, Schreiber LRN, Christenson G, Derbyshire K, Grant JE. Skin picking disorder in university students: health correlates and gender differences. Gen Hosp Psychiatry 2013; 35: 168-73.

9 Stein DJ, Chamberlain SR, Fineberg N. An A-B-C model of habit disorders: hair-pulling, skin-picking, and other stereotypic conditions. CNS Spectr 2006; 11: 824-7.

10 Lovato L, Ferrão YA, Stein DJ, Shavitt RG, Fontenelle LF, Vivan A, et al. Skin picking and trichotillomania in adults with obsessive-compulsive disorder. Compr Psychiatry 2012; 53: 562-8.

11 Monzani B, Rijsdijk F, Harris J, Mataix-Cols D. The structure of genetic and environmental risk factors for dimensional representations of DSM-5 obsessive-compulsive spectrum disorders. JAMA Psychiatry 2014; 71: 182-9.

12 Grant JE, Odlaug BL, Hampshire A, Schreiber LR, Chamberlain SR. White matter abnormalities in skin-picking disorder: a diffusion tensor imaging study. Neuropsychopharmacology 2013; 38: 763-9.

13 Chamberlain SR, Hampshire A, Menzies LA, Garyfallidis E, Grant JE, Odlaug $B L$, et al. Reduced brain white matter integrity in trichotillomania: a diffusion tensor imaging study. Arch Gen Psychiatry 2010; 67: 965-71.

14 Chamberlain SR, Menzies LA, Fineberg NA, Del Campo N, Suckling J, Craig K, et al. Grey matter abnormalities in trichotillomania: morphometric magnetic resonance imaging study.

Br J Psychiatry 2008; 193: 216-21.

15 Menzies L, Chamberlain SR, Laird AR, Thelen SM, Sahakian BJ, Bullmore ET. Integrating evidence from neuroimaging and neuropsychological studies of obsessive-compulsive disorder: the orbitofronto-striatal model revisited. Neurosci Biobehav Rev 2008; 32: 525-49.

16 Chamberlain SR, Fineberg NA, Blackwell AD, Clark L, Robbins TW, Sahakian BJ. A neuropsychological comparison of obsessive-compulsive disorder and trichotillomania. Neuropsychologia 2007; 45: 654-62.

17 Tükel R, Gürvit H, Ertekin BA, Oflaz S, Ertekin E, Baran B, et al. Neuropsychological function in obsessive-compulsive disorder. Compr Psychiatry 2012; 53: 167-75.

18 Kashyap H, Kumar JK, Kandavel T, Reddy YC. Neuropsychological functioning in obsessive-compulsive disorder: are executive functions the key deficit? Compr Psychiatry 2013; 54: 533-40.

19 Delorme R, Goussé V, Roy I, Trandafir A, Mathieu F, Mouren-Siméoni MC, et al. Shared executive dysfunctions in unaffected relatives of patients with autism and obsessive-compulsive disorder. Eur Psychiatry 2007; 22: 32-8.

20 Cavedini P, Zorzi C, Piccinni M, Cavallini MC, Bellodi L. Executive dysfunctions in obsessive-compulsive patients and unaffected relatives: searching for a new intermediate phenotype. Biol Psychiatry 2010; 67: 1178-84.

21 van den Heuvel OA, Veltman DJ, Groenewegen HJ, Cath DC, van Balkom AJ, van Hartskamp J, et al. Frontal-striatal dysfunction during planning in obsessive-compulsive disorder. Arch Gen Psychiatry 2005; 62: 301-9.

22 Arnold LM, Mutasim DF, Dwight MM, Lamerson CL, Morris EL, McElroy SL. An open clinical trial of fluvoxamine treatment of psychogenic excoriation. J Clin Psychopharmacol 1999; 19: 15-8.

23 Grant JE, Odlaug BL, Kim SW. Lamotrigine treatment of pathologic skinpicking: an open-label study. J Clin Psychiatry 2007; 68: 1384-91.

24 First MB, Spitzer RL, Gibbon M (1995). Structured Clinical Interview for DSM-IV Axis I Disorders. Biometrics Research Department, New York State Psychiatric Institute.

25 de Ruiter MB, Veltman DJ, Goudriaan AE, Oosterlaan J, Sjoerds Z, van den Brink W. Response perseveration and ventral prefrontal sensitivity to reward and punishment in male problem gamblers and smokers. Neuropsychopharmacology 2009; 34: 1027-38.

26 Kräplin A, Bühringer G, Oosterlaan J, van den Brink W, Goschke T, Goudriaan AE. Dimensions and disorder specificity of impulsivity in pathological gambling. Addict Behav 2014; 39: 1646-51. 
27 Nombela C, Rowe JB, Winder-Rhodes SE, Hampshire A, Owen AM, Breen DP, et al. Genetic impact on cognition and brain function in newly diagnosed Parkinson's disease: ICICLE-PD Study. Brain 2014; 137: 2743-58.

28 Williams-Gray $\mathrm{CH}$, Hampshire A, Robbins TW, Owen AM, Barker RA. Catecho O-methyltransferase Val158Met genotype influences frontoparietal activity during planning in patients with Parkinson's disease. J Neurosci 2007; 27: 4832-8

29 Hampshire A, MacDonald A, Owen AM. Hypoconnectivity and hyperfrontality in retired American football players. Sci Rep 2013; 3: 2972.

30 Kempton MJ, Ettinger U, Foster R, Williams SC, Calvert GA, Hampshire A, et al. Dehydration affects brain structure and function in healthy adolescents. Hum Brain Mapp 2011; 32: 71-9.

31 Bennett $\mathrm{CM}$, Wolford GL, Miller MB. The principled control of false positives in neuroimaging. Soc Cogn Affect Neurosci 2009; 4: 417-22.
32 Brett $\mathrm{M}$, Anton J, Valabregue $\mathrm{R}$, Poline JB. Region of interest analysis using an SPM toolbox (abstract). Neuroimage 2002; 16 (suppl 1): 1140-1 (http:// matthew.dynevor.org/_downloads/marsbar_abstract.pdf).

33 Brennan BP, Rauch SL, Jensen JE, Pope HG Jr. A critical review of magnetic resonance spectroscopy studies of obsessive-compulsive disorder. Biol Psychiatry 2013; 73: 24-31

34 Borairi S, Dougherty DD. The use of neuroimaging to predict treatment response for neurosurgical interventions for treatment-refractory major depression and obsessive-compulsive disorder. Harv Rev Psychiatry 2011; 19: 155-61.

35 Tolin DF, Stevens MC, Villavicencio AL, Norberg MM, Calhoun VD, Frost RO, et al. Neural mechanisms of decision making in hoarding disorder. Arch Gen Psychiatry 2012; 69: 832-41.

36 Gawryluk JR, Mazerolle EL, Brewer KD, Beyea SD, D'Arcy RC. Investigation of fMRI activation in the internal capsule. BMC Neurosci 2011; 12: 56.

\section{psychiatry in history}

\section{An 18th-century view of demonomania. I: Classification}

\section{Fiona Subotsky}

Within the archives of the Royal College of Psychiatrists were recently discovered about 80 MD theses on psychiatric topics dating from the 18th century. They are in Latin, come from locations across Europe and must have been specially collected, although unfortunately the donor is not recorded.

I looked more closely at a thesis entitled 'Dissertatio inauguralis practico-medica de Daemonomania et variis ejus speciebus' ('Inaugural medical dissertation on Demonomania and its various subclasses'). The author, Martinus Martini from Saxon Transylvania, presented this at the University of Vienna in 1782. His tone is that of a young man who, while presenting a sensational topic in an apparently challenging way, makes considerable use of earlier sources and expects the approval of at least some of his seniors.

Martini explains that the name 'Demonomania' was given to conditions in which 'a Demon is said to have exercised its force in various ways on men and other animate and inanimate things'. He distinguishes demonomania from other forms of insanity first by their psychopathology:

Demoniacs rave constantly ... about diabolical supernatural things only. Maniacs are disturbed first by one then by another object . . .

and also sociologically:

The majority with Demonomania are crude, untaught, poor and servile; but with melancholia belong to a group more delicate and tender, educated, clever and prosperous.

He classifies the condition into three: first 'True Demonomania', the existence of which he questions; second 'Feigned Demonomania' in which he includes witches, vampires, religious or political fanaticism, and amok due to opium; third, 'Falsely Ascribed Demonomania', which includes causes such as worms, hysteria, the 'Polish Plait' and 'cardiac'.

Martini's structure and many of his descriptions are essentially taken from Francois Boissier de Sauvages de Lacroix (1706-1767). The latter was professor of physiology and pathology, and later also of botany, at Montpellier University. He was a friend of Linnaeus, whose classification system Sauvages developed as a pattern for medical diagnosis in his Nosologia Methodica, published in 1763. This classification of 2400 medical diseases was divided into 10 'classes', one of which comprised the bulk of the mental diseases, divided into four 'orders' and 23 'genera', one of which was Demonomania. Linnaeus also classified diseases in Genera Morborum (1763) and included Demonomania, which he characterised as a 'chronic insanity, partial, furious, meticulous and concerning demons', but does not include vampirism.

In Britain, William Cullen (1710-1790), professor at Edinburgh, disagreed in his Synopsis Nosologia Methodica (1769), remarking that:

I do not allow that there is any true daemonomania; and, in my opinion, the species recorded by Sauvages are either, 1. Species of melancholia or mania 2. Diseases falsely referred by spectators to the power of demons 3. Feigned diseases, or 4. Diseases partly real (as 2.), or partly feigned (as 3.).

This is similar to Martini's grouping although Martini does not reference Cullen.

Cullen's scheme influenced Philippe Pinel, who developed his own classification of what he called mental alienation (Treatise on Insanity, 1806). He did not use the terminology of demonomania except in reference to the belief of religious rituals, which he set largely in the unenlightened past:

In some instances it was believed that the intellectual faculty was ... deranged by the malignant influence of a demon: in others, where the change of character was more evident and more complete, an actual exchange of the indwelling soul was imagined to have taken place ...

Martini himself is entirely sceptical of the supernatural, and offers alternative biological and psychological explanations in some detail, especially in the case of vampirism - but that is another story . . .

The British Journal of Psychiatry (2016) 208, 174. doi: 10.1192/bjp.bp.114.1612 